\title{
Granular flows on a dissipative base
}

\author{
Michel Y. Louge,,${ }^{1, *}$ Alexandre Valance,${ }^{2, \dagger}$ Paul Lancelot,,${ }^{3, \dagger}$ Renaud Delannay, ${ }^{2}$ and Olivier Artières ${ }^{4, \S}$ \\ ${ }^{1}$ Cornell University, Ithaca, New York 14853, USA \\ ${ }^{2}$ Universite de Rennes 1, 35042 Rennes Cedex, France \\ ${ }^{3}$ Faculty of Aerospace Engineering Delft University of Technology 2629 HS Delft, The Netherlands \\ ${ }^{4}$ TenCate GeoSynthetics 9, rue Marcel Paul, F-95873 Bezons CEDEX, France
}

(Received 18 February 2015; published 10 August 2015)

\begin{abstract}
We study inclined channel flows of sand over a sensor-enabled composite geotextile fabric base that dissipates granular fluctuation energy. We record strain of the fabric along the flow direction with imbedded fiber-optic Bragg gratings, flow velocity on the surface by correlating grain position in successive images, flow thickness with the streamwise shift of an oblique laser light sheet, velocity depth profile through a transparent side wall using a high-speed camera, and overall discharge rate. These independent measurements at inclinations between $33^{\circ}$ and $37^{\circ}$ above the angle of repose at $32.1 \pm 0.8^{\circ}$ are consistent with a mass flow rate scaling as the $3 / 2$ power of the flow depth, which is markedly different than flows on a rigid bumpy boundary. However, this power changes to $5 / 2$ when flows are forced on the sand bed below its angle of repose. Strain measurements imply that the mean solid volume fraction in the flowing layer above the angle of repose is $0.268 \pm 0.033$, independent of discharge rate or inclination.
\end{abstract}

DOI: 10.1103/PhysRevE.92.022204

PACS number(s): 45.70.Mg, 45.70.Vn, 45.50.-j, 45.70.Ht

\section{INTRODUCTION}

Inclined flows of granular materials have attracted attention from the physics and engineering communities because of their importance in industry and earth science. Experiments have mainly focused on wall-bounded flows on a hard base, either flat and frictional [1,2], or covered with bumps with size on the order of flow particles [1,3-5]. Fewer studies have considered flows on erodible surfaces made up of loose grains [6-13], despite their prevalence in natural settings, such as the leeward avalanche face of an advancing sand dune [14], self-channeling flows on volcanic slopes [15,16], or, if an interstitial liquid is present, debris flows on hills [17] or perhaps other planets [18]. The character of an erodible base is that it readily dissipates granular fluctuation kinetic energy [19], thereby reducing granular viscosity and other granular transport coefficients [20].

More generally, global flow characteristics are affected by the nature of the basal boundary. Flows of moderate thickness on a rigid bumpy base have a granular viscosity proportional to the square root of the distance to the free surface, and their velocity vanishes at the base. Consequently, they are sheared through their depth and exhibit a "Bagnold" profile with mass flow rate proportional to the $5 / 2$ power of the depth [21]. In the absence of side walls, grain flows released on a rigid bumpy base expand laterally until parallel levées form naturally with separation rising with the imposed upstream mass flow rate $[15,16]$.

\footnotetext{
*michel.louge@cornell.edu

†alexandre.valance@univ-rennes1.fr

${ }^{\ddagger}$ p.m.g.j.lancelot@tudelft.nl

§o.artieres@TENCATE.COM
}

Published by the American Physical Society under the terms of the Creative Commons Attribution 3.0 License. Further distribution of this work must maintain attribution to the author(s) and the published article's title, journal citation, and DOI.
In contrast, flow velocity profiles above a flat, frictional plane at moderate angles of inclination exhibit a large velocity relative to the base, known as "slip." A balance of angular momentum in the thin basal layer of rolling grains yields a mean slip proportional to the square root of flow depth, therefore producing an overall mass flow rate rising as the $3 / 2$ power of the depth [22]. Granular flows on flat walls also feature more complex structure at high acceleration [2] or speed [23]. In the latter case, the power of depth is closer to $5 / 4$ [24].

Flows over an erodible, energy-dissipating heap behave altogether differently. If nearby frictional side walls confine them closely in the transverse direction, flows of sufficient mass flow rate can reach an inclination $\alpha$ that is much steeper than the angle of repose. Because friction on side walls is a crucial part of the force balance in these "sidewall-stabilized heaps" (SSHs), the ratio of flow depth to side wall distance $W$ is a linear function that grows with $\tan \alpha$ [9]. As a result, $W$ is the principal length scale for flow depth and mass flow rate and for depth profiles of velocity and solid volume fraction [25]. Nonetheless, streamwise velocity profiles remain surprisingly uniform in the transverse direction $[9,26]$.

On an erodible bed of glass beads at lower inclinations, the measurements of Jop et al. [10] suggest that side walls affect the transverse profiles of streamwise velocity, albeit within a relatively small characteristic distance from the wall that decreases sharply with increasing $\alpha$. However, on erodible slopes like the leeward face of a sand dune, it is common to observe granular flows with diverse widths but similar speed. This suggests that lateral boundaries, if located far enough away, do not matter to the local solid flux, insofar as the flow widens spontaneously when the overall imposed granular mass flow rate is increased [16].

As stated in Ref. [27], natural granular flows on deep erodible heaps without side walls are observed somewhere between the "angle of initial yield of the material" $\alpha_{a}$, which varies with volume fraction of the deposited grains, and the smaller, nearly constant "residual angle after shearing" $\alpha_{r}$ that 
is reached as the flow stops. In the laboratory, $\alpha_{a}$ is measured by inclining the surface of a granular bed and noting the angle at which an avalanche triggers spontaneously. The residual angle $\alpha_{r}$ is then obtained by recording the inclination of the free surface once the avalanche has run its course. Because avalanches return natural heaps to this residual inclination, $\alpha_{r}$ is also known as the angle of repose.

To approximate flows on natural heaps at several inclinations, in Ref. [28] glass beads were released on a plane covered with a dissipative felt cloth in the laboratory. Transient flows of evolving width were established for thicknesses in the range $h_{\text {stop }}<h<h_{\text {start }}$, where $h_{\text {start }}$ and $h_{\text {stop }}$ were, respectively, values of $h$ at which the flow starts and stops.

In this context, we investigated flows on a dissipative base with side walls far enough to matter little to the transverse profile of streamwise velocity. Our objective was to establish the dominant length scale for such wide flows, which are ubiquitous on the leeward face of sand dunes, and to address basic questions. With distant side walls, what scale determines the mass flow rate? Like $h_{\text {stop }}$, does it depend on inclination? Is the mean solid volume fraction in the flowing layer as low as in a SSH? Can such a volume fraction be reliably measured?

To mimic basal fluctuation energy dissipation without staging a deep sand pile, we ran these flows on a sensor-enabled composite geotextile laid upon a hard inclined plane, similar to the felt cloth used in Ref. [28]. A benefit of this layout was to bind optical fiber Bragg gratings [29] (FBGs) with the geotextile to record its local strain in the streamwise direction. An analysis of the geotextile's elasticity then yielded the integrated solid volume fraction in the flowing layer, which was called mass holdup in Ref. [1].

Additional records of overall discharge mass flow rate and velocity confirmed that flows on an erodible base behave differently than those on hard surfaces. We found that this apparatus, although possessing a much larger width, exhibited similar mean solid volume fraction and velocity profiles to SSHs $[9,25]$. However, because side wall distance could not act as the dominant characteristic length, our flows had mass flow rates and velocities scaling with fractional powers of the flow thickness.

We begin this article with a brief description of the apparatus. We then derive how measurements of geotextile microstrain yield mass holdup. Finally, we exploit the data to establish how mass flow rate, speed, and mean volume fraction scale with inclination and flow depth.

\section{APPARATUS}

We discharged "NE 34" sand of material density $\rho_{s} \simeq$ $2650 \mathrm{~kg} / \mathrm{m}^{3}$ produced by Sibelco from a reservoir onto an inclined plane of length $L=130 \mathrm{~cm}$ bounded by transparent side walls separated by $W=28.5 \mathrm{~cm}$ (Fig. 1). Our channel was significantly wider relative to the flow thickness $\Delta h$ $(22<W / \Delta h<140)$ than the experiments of Jop et al. $(0.7<$ $W / \Delta h<15)[10]$.

Based on the AFNOR X.11-507 sieving protocol, moments of the particle size distribution (PSD) by mass were $\bar{d} \simeq$ $354 \mu \mathrm{m},\left(\overline{d^{2}}\right)^{1 / 2} \simeq 366 \mu \mathrm{m}$, and $\left(\overline{d^{3}}\right)^{1 / 3} \simeq 376 \mu \mathrm{m}$, in which the overbar denotes averaging over the PSD. Thus, the standard deviation of $d$ was $\pm 92 \mu \mathrm{m}$. For this sand we measured an

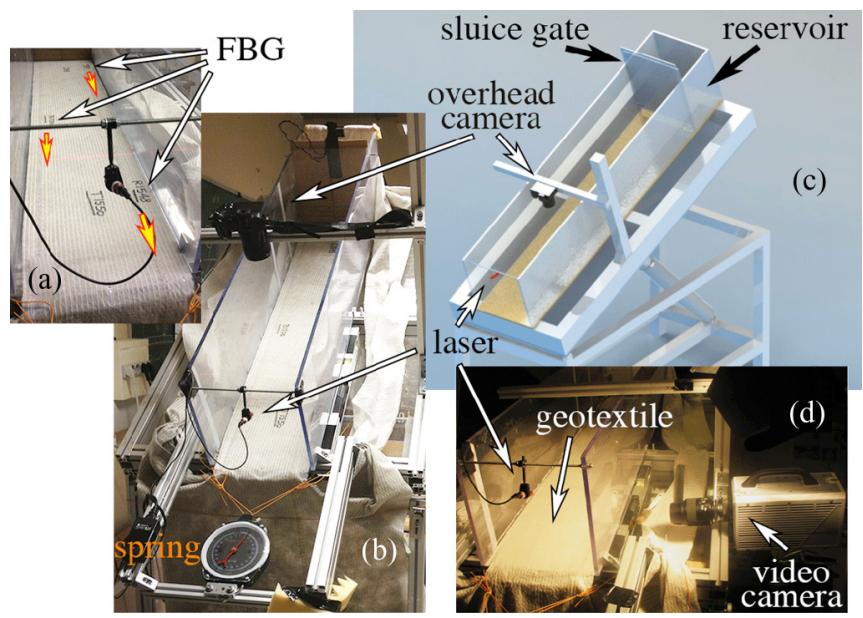

FIG. 1. (Color online) Apparatus. (a) Position and orientation of the three FBGs. (b) Overview showing the bare geotextile laid at the base of the channel and the spring that tensions it at the exit. (c) Sketch showing reservoir, sluice gate, laser profiler, and overhead camera. (d) Detail near the exit showing the high-speed video camera trained to observe the flow through one of the transparent side walls.

angle of repose $\alpha_{r}=32.11 \pm 0.77^{\circ}$ and an angle of initial yield $\alpha_{a}=37.4 \pm 2.5^{\circ}$. (Consistent with the observations in Ref. [27], $\alpha_{a}$ exhibited greater scatter than $\alpha_{r}$.)

In this facility, sand was released through a sluice gate of adjustable opening onto a nonwoven sensor-enabled composite geotextile manufactured by TenCate Geosynthetics. The sensor-enabled geotextile was laid on the metal base of the chute and firmly anchored on a straight line across the width of the channel just inside the reservoir. It consisted of a needle-punched nonwoven base reinforced with longitudinal polyester yarns that conferred it a constant stiffness. Therefore, the force $f$ in a unit width was related to the local strain $\epsilon$ of the geotextile through

$$
f=\kappa \epsilon,
$$

where $\kappa \simeq 317 \pm 32 \mathrm{kN} / \mathrm{m}$. In the same way as the reinforcing yarn, two optical fibers were firmly sewn to the geotextile along the flow direction $x$. They contained FBGs consisting of periodic variations in the refractive index over a short fiber length [29]. Each FBG reflected a narrow range of wavelengths $\lambda$ while transmitting all others. Any increment $d \epsilon$ in local strain imposed on the fiber increased the periodic spacing, thus raising the reflected wavelength by a corresponding relative increment

$$
d \lambda / \lambda=C_{s} d \epsilon .
$$

A detector recorded the change in wavelength relative to its nominal value, thus yielding the incremental strain. For the Tencate GeoDetect-S system that we used, $C_{s} \simeq 0.78410^{-6}$. Although FBGs are also sensitive to temperature $T$ through an additional term $C_{T} d T$ in Eq. (2) with $C_{T} \simeq 6.5410^{-6}{ }^{\circ} \mathrm{K}^{-1}$, we did not take variations of $T$ into account, since our measurements relied upon differences between strains recorded during and after experiments, and temperature varied negligibly in each test, as evidenced by signals from a similar FBG laid below the geotextile but unbound to its fabric. 
The sensing geotextile strip was positioned so the two parallel optical fibers rested on opposite sides of the channel centerline at a transverse distance $y= \pm 10 \mathrm{~cm}$ [Fig. 1(a)]. The right optical fiber at $y=10 \mathrm{~cm}$ contained two FBGs of nominal wavelengths $\lambda=1553$ and $1548 \mathrm{~nm}$ at respective streamwise distances $x \simeq 17$ and $117 \mathrm{~cm}$ from the upstream anchor below the sluice gate. The left fiber at $y=-10 \mathrm{~cm}$ had a single FBG with $\lambda=1550 \mathrm{~nm}$ at $x=67 \mathrm{~cm}$. All three FBGs were far enough from side walls $(W / 2-|y|) \simeq 120 \bar{d}$ that they were unaffected by their presence.

We measured the coefficient of static friction $\mu$ between composite geotextile and rigid chute by laying on the Plexiglas base a plastic brick covered with the geotextile and inclining the chute until the brick started to slide. The corresponding angle $\alpha_{g}$ then yielded $\mu=\tan \alpha_{g}=0.3620 \pm 0.0020$. Because the "filtration opening size" $\sim 100 \mu \mathrm{m}$ of this geotextile (i.e., the diameter of its largest opening) was smaller than the smallest sand particle, no dust accumulated between its fabric and the chute base, and therefore $\mu$ did not change.

A Photron high-speed camera with $1024 \times 1024$ pixel resolution acquired images of the flow through the right Plexiglas side wall at 1500 frames per second (fps) with a Nikon macrozoom lens of $85 \mathrm{~mm}$ focal length. Differentialparticle-image-velocimetry (DPIV) software then inferred the velocity of individual grains, ignoring the contribution of immobile imperfections on the side wall by truncating velocity statistics below $2 \mathrm{~cm} / \mathrm{s}$. Meanwhile, an overhead digital camera captured images of the moving free surface at $60 \mathrm{fps}$ for all angles of inclinations considered. The DPIV software satisfactorily handled surface grain speeds $<80 \mathrm{~cm} / \mathrm{s}$ at this relatively low frame rate. Manual observations of the displacement of recognizable grains was used for higher speeds.

The overhead camera also viewed the intersection of a plane of laser light inclined at an angle $\beta \simeq 27^{\circ}$ steeper than the base, as it created a bright transverse red line perpendicular to the side walls by intersecting the bare geotextile or the sand surface. The observed streamwise shift $\delta$ of the intersecting line with or without sand then yielded the thickness $h=\delta \tan \beta$ of the sand bed, whether the latter was mobile or not. At their relatively small inclinations, our flows were hardly agitated, producing a low granular temperature $\Theta_{s}$ at the free surface. Because particle number density in the nearly collisionless ballistic layer above the flow decayed exponentially with altitude on a length scale $\Theta_{s} / g$, where $g$ is the acceleration of gravity [30], the ballistic layer was very thin. Therefore, the laser produced an accurate measurement of $h$ that was uncorrupted by fuzziness typically seen in steeper flows [25]. We carried out this measurement at several distances along the flow without discerning any change in thickness. Thickness data reported here were obtained at $1 \mathrm{~m}$ from the sluice gate.

Finally, as the sluice gate opened to empty out the reservoir, we recorded the sand mass discharged downstream to a bucket standing on a digital balance by filming the latter at $30 \mathrm{fps}$.

In short, our apparatus yielded independent measurements of grain velocity $u$ across the free surface and along depth at the side walls, flow depth $h$ above the geotextile, discharge mass flow rate $\dot{m}$, and time history of geotextile strain $\epsilon$ at three positions along the flow. While the first three measurements were relatively straightforward, the interpretation of the strain required an analysis of how the geotextile sensing strip responded to the presence of the entire sand bed flowing between its anchor and the downstream chute discharge. As the next section outlines, this analysis yielded an accurate record of the mean solid volume fraction in the flowing layer.

\section{GEOTEXTILE STRAIN ANALYSIS}

In this experiment, a spring exerts a uniform traction on the geotextile across the entire downstream width where the flow is discharged. The geotextile is then slightly stretched between its upstream anchor and the discharge by a tensile force $f$ per unit width. It is also subject to three additional forces: the sand weight that it supports, the reaction force exerted by the rigid base of the chute, and its own weight.

A balance of forces on a unit width and along an infinitesimal length $d x$ of the geotextile is shown in Fig. 2. Far away from walls $(W / 2-|y| \gg \ell)$, the balance is independent of $y$, and it yields a differential equation governing the evolution of $f$ along $x$,

$$
\frac{\partial f}{\partial x}=-(\tan \alpha-\mu) g \cos \alpha(\omega+H),
$$

where $\mu$ is the friction coefficient between geotextile and base, $\omega$ is geotextile surface density, and $H$ is the mass of grains
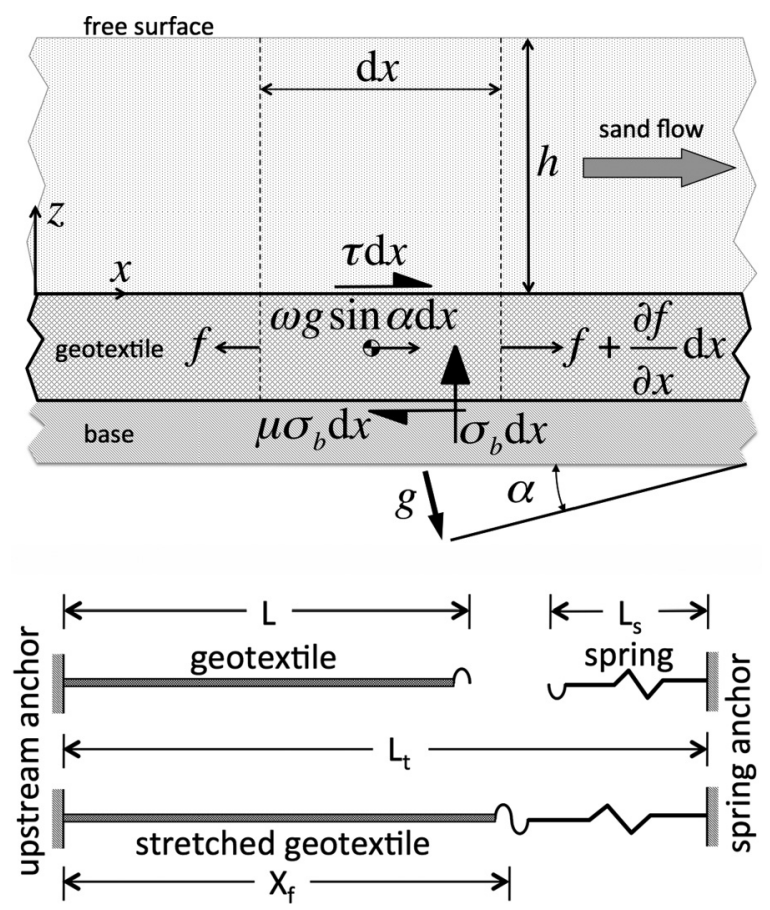

FIG. 2. Forces and lengths. Top: Force balance on a unit width of the geotextile in the flow direction $x$ at the inclination $\alpha$ along an infinitesimal length $d x$. The projection of geotextile weight along $x$ is $\omega g \sin \alpha d x$. Sand of thickness $h$ flowing or resting overhead exerts a downhill shear force $\tau d x=\rho_{s} g \sin \alpha d x \int_{z=0}^{h} v d z$. The combined weight of sand and geotextile then presses on the base with normal force $\sigma_{b} d x=\left(\omega+\rho_{s} \int_{z=0}^{h} v d z\right) g \cos \alpha d x$. This gives rise to an uphill shear force $\mu \sigma_{b} d x$. Bottom: Lengths of geotextile and spring at rest (top) and stretched after being joined (bottom). 
bearing on a unit area of geotextile,

$$
H \equiv \rho_{s} \int_{z=0}^{h} v d z .
$$

This quantity is related to the dimensionless "mass holdup" $H^{\dagger} \equiv H /\left(\rho_{s} d\right)$ that the authors of Ref. [1] obtained by integrating the solid volume fraction $v$ as it evolved along the depth $z$. Defining the net longitudinal acceleration

$$
A \kappa \equiv(\tan \alpha-\mu) g \cos \alpha,
$$

Eqs. (1) and (3) integrate to a strain

$$
\epsilon=-A v(x)+b-1,
$$

where $b$ is a constant to be determined, and

$$
v(x) \equiv \int_{x^{\prime}=0}^{x}\left[H\left(x^{\prime}\right)+\omega\right] d x^{\prime}
$$

represents the combined mass of sand and geotextile from the anchor to $x$.

Meanwhile, focusing on material points bound to the geotextile, we define the stretched distance $X$ of one such point to the anchor. Another such point initially at an unstretched infinitesimal distance $d x$ downstream is now located at $X+(\partial X / \partial x) d x=X+(1+\epsilon) d x$ after stretching. Therefore, upon placing the origin at the upstream geotextile anchor on the channel centerline, the distance of any geotextile-bound point to the anchor satisfies

$$
\frac{\partial X}{\partial x}=(1+\epsilon)
$$

with initial condition $X=0$ at $x=0$. Then Eqs. (6)-(8) yield

$$
X=-A \int_{x^{\prime}=0}^{x} v\left(x^{\prime}\right) d x^{\prime}+b x .
$$

The geotextile of rest length $L$ is tensioned by a spring of constant $k_{s}$ and rest length $L_{s}$ firmly held at a distance $L_{t}$ from the top anchor such that

$$
F=k_{s}\left(L_{t}-L_{s}-X_{f}\right),
$$

where $X_{f}=X(L)$ is the length of the geotextile stretched by the combination of spring, sand, and geotextile weight (Fig. 2). For convenience, we define an effective spring constant for the geotextile

$$
k_{g} \equiv \frac{\kappa W}{L} .
$$

Then the force balance at spring's end $(x=L)$ is

$$
F=W f=\kappa W \epsilon=\kappa W[-A v(L)+b-1],
$$

from which we extract the constant $b$ and deduce strain along the geotextile

$$
\epsilon=A\left[v_{m}-v(x)\right]+\epsilon_{m} .
$$

In this equation we define

$$
\epsilon_{m} \equiv\left(\frac{k_{s}}{k_{s}+k_{g}}\right) \frac{\Delta L}{L},
$$

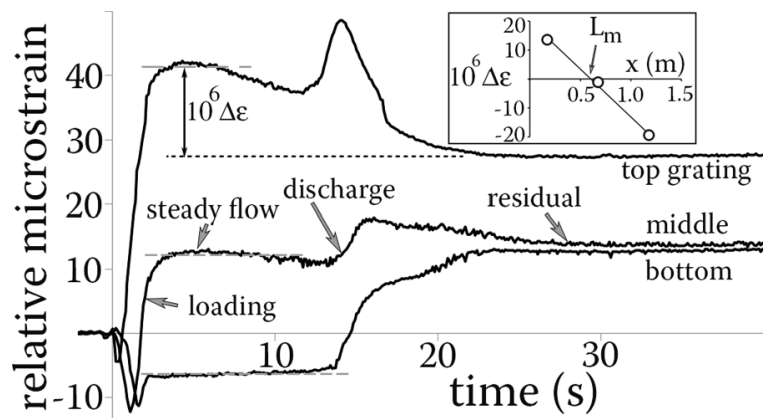

FIG. 3. Typical microstrain time histories from the top, middle, and bottom FBGs with $\tan \alpha \simeq 0.70$ at a steady flow depth $\left(h_{\text {stop }}-\right.$ $h) / \bar{d} \simeq 16.2$. The abscissa is time in seconds after opening the sluice gate. The ordinate is $10^{6} \times \epsilon$, translated to superimpose the three signals for $t<0$. (The three strains have widely different magnitude, but what matters is their relative change $\Delta \epsilon$.) The loading, steady flow, and discharge phases are marked on the signal of the middle FBG, along with its residual strain after the flow stops. Gray dashed lines show the period of steady strain, lasting at least $4 \mathrm{~s}$. The microstrain differences $10^{6} \Delta \epsilon$ between these steady plateaus at a flow depth $h$ and the residual strain at $h_{\text {stop }}$ (dotted lines) are plotted in the inset vs FBG distance $x$ to the upstream geotextile anchor in meters.

where $\Delta L \equiv L_{t}-\left(L_{s}+L\right)$ is the combined stretched lengths of spring and geotextile, and

$$
v_{m} \equiv \frac{k_{g} v(L)+k_{s} \mathbb{V}_{L}}{k_{g}+k_{s}},
$$

where

$$
\mathbb{V}_{L} \equiv \frac{1}{L} \int_{x^{\prime}=0}^{L} v\left(x^{\prime}\right) d x^{\prime} .
$$

Figure 3 shows strain time histories of the three Bragg gratings for a typical experiment. As sand is released on the geotextile, the three FBGs initially undergo a loading phase, during which oncoming sand weight first compresses the geotextile ahead of the sharp advancing granular front, thus causing the three FBGs to experience a rapidly decreasing strain. Then, passage of the front above each FBG abruptly reverses the trend, producing an increasing strain due to the integrated weight of sand downhill of the FBG. The loading phase ends when the oncoming sand front washes over the exit. Afterwards, with enough sand in the reservoir, the flow adopts uniform steady thickness and holdup, which are controlled by the opening of the sluice gate, thus producing for a few seconds plateaus of the FBG signal marked by horizontal dashed lines in Fig. 3.

The discharge phase begins as the reservoir no longer contains enough sand to cover the entire sluice gate, thus producing a flow thickness that decreases slowly with time but remains nearly uniform along the chute. For several seconds, the three FBGs thus record signals that are consistent with a decreasing, albeit uniform flow thickness. When the reservoir is completely exhausted, sand first comes to a halt upstream, while other grains are progressively offloaded with a streamwise velocity that is faster on the surface than below, consistent with the depth velocity profile. In this process, each FBG records a reverse, but more gradual, strain time history than upon loading. First, it experiences a rising strain, as more 
sand covers the geotextile downstream of its position than upstream. Then its strain gradually reaches a steady value consistent with the depth $h_{\text {stop }}$ of grains resting uniformly along the whole chute.

An alternative to our FBGs is a local strain gauge platform, which can yield useful information on local basal shear stress if the latter is intense enough to yield a high signal-to-noise ratio [31-33]. However, such strain is difficult to interpret in granular flows with a relatively thin flowing layer. Instead, because our geotextile-bound FBGs are sensitive to sand weight integrated from the grating to the chute discharge where the geotextile is tensioned, their recorded strain is greater and less subject to noise. However, FBG strain does not distinguish local details, and its interpretation is most meaningful when the flow is steady and fully developed (SFD). In that case, because the noninvasive FBG is tightly bound to the geotextile, and because its interferometric strain requires no calibration, its signal is reliable, strong, and precise [34].

With a SFD flow, the mass holdup $H$ is invariant in time and along $x, v(x)=(H+\omega) x, \mathbb{V}_{L}=L(H+\omega) / 2$, and $v_{m}=\left[\left(2 k_{g}+k_{s}\right) /\left(k_{g}+k_{s}\right)\right](H+\omega) L / 2$. Then strain along the geotextile has the form

$$
\epsilon=A(H+\omega)\left(L_{m}-x\right)+\epsilon_{m},
$$

where

$$
L_{m} \equiv \frac{L}{2}\left(\frac{2 k_{g}+k_{s}}{k_{g}+k_{s}}\right)
$$

is the distance from the top anchor where strain is invariant and equal to $\epsilon_{m}$. Note that this strain "focal point" is always in the second half of the geotextile, $(L / 2)<L_{m}<L$.

As shown in Fig. 3, we form the difference $\Delta \epsilon$ between discernible strain plateaus from the three FBGs and their respective strain residuals. From Eq. (17), we find

$$
\Delta \epsilon=A\left(H-H_{\text {stop }}\right)\left(L_{m}-x\right) .
$$

This result shows that, when $H$ becomes $H_{\text {stop }}$ as flow comes to an end, strain recorded with a FBG at $x<L_{m}$ increases $(\Delta \epsilon>0)$; however, it decreases $(\Delta \epsilon<0)$ for $x>L_{m}$. This is evident in Fig. 3, where $\Delta \epsilon>0$ for the top FBG, but $<0$ for the bottom. Note that $\Delta \epsilon$ is independent of surface density of the geotextile itself.

As Eq. (17) shows, strain can also become negative near the exit if

$$
A(H+\omega)\left(L-L_{m}\right)>\epsilon_{m} .
$$

Practically, this would occur when sand thickness and/or inclination are excessive, or when the spring is not tensioned enough. In that case, the geotextile experiences compression (i.e., bulging) $\forall x>L_{m}+\epsilon_{m} /[A(H+\omega)]$. The inset of Fig. 3 and Eq. (18) also suggest that, because $L_{m} \simeq L / 2$, our tests were carried out in the limit where the spring is much stiffer than the geotextile matrix, $k_{s} \gg k_{g}$. As Eq. (15) shows, $v_{m} \simeq \mathbb{V}_{L}$ in that limit.

As Eq. (19) reveals, a graph of $\Delta \epsilon$ vs FBG location (inset in Fig. 3) yields a slope $A\left(H-H_{\text {stop }}\right)$. Because the bed located beneath a distance $\left(h-h_{\text {stop }}\right)$ from the free surface is nearly immobile, it condenses at a relatively large solid volume fraction $v \simeq v_{c}$ that is invariant with depth and remains unchanged as the flow stops, as experiments [19]

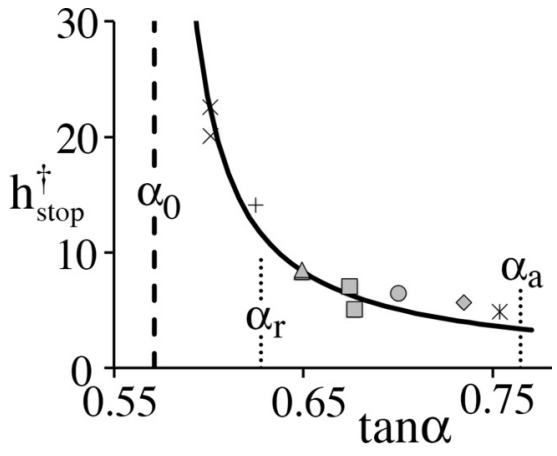

FIG. 4. Residual depth of sand on the geotextile $h_{\text {stop }}^{\dagger} \equiv h_{\text {stop }} / \bar{d}$, dimensionless with mean grain diameter, vs tangent of the angle of inclination $\alpha$. The solid line is the least-squares fit in Eq. (22). The vertical lines mark tangents of the asymptotic stop angle $\alpha_{0}$, the angle of repose $\alpha_{r}$, and the angle of spontaneous avalanching $\alpha_{a}$. Identical symbols are used in subsequent figures to identify inclination.

have suggested for the SSH. Therefore, the difference in mass holdup cancels the contribution of $v_{c}$, and it depends only on the integrated mean solid volume fraction $\bar{v}$ within the flowing layer,

$$
H-H_{\text {stop }}=\rho_{s} \int_{z=h_{\text {stop }}}^{h} v d z \equiv \rho_{s}\left(h-h_{\text {stop }}\right) \bar{\nu} .
$$

Then the FBG determination of $H-H_{\text {stop }}$ lets us infer $\bar{v}$ from the slope of $H-H_{\text {stop }}$ versus $\left(h-h_{\text {stop }}\right)$ measured using the laser sheet described in Sec. II. Although the actual value of $v_{c}$ matters little to our differential measurement of $\bar{v}$, we recorded it by combining a laser record of deposited sand thickness with a balance of sand mass released at the reservoir and discharged at the exit. In good agreement with numerical simulations [25], we found $v_{c}=0.600 \pm 0.015$ and $0.602 \pm 0.011$ at $\alpha=31.5^{\circ}$ and $32.5^{\circ}$, respectively.

\section{RESULTS}

In the conduct of granular flow observations, it is challenging to establish how mass flow rate scales with mass holdup, angle of inclination and residual depth, if $\dot{m}, h$, $h_{\text {stop }}$, and $\alpha$ are the only recorded variables. To confirm the scaling, we found it useful to record surface velocity and mass holdup independently, since mass flow rate and velocity differ by a factor on the order $\left(H-H_{\text {stop }}\right)$. In this quest, geotextile and FBG were crucial for establishing the relation between flow depth and mass holdup in Eq. (21). This section summarizes results, all quoted with error bars at $95 \%$ confidence. Dimensionless quantities are denoted with a dagger superscript.

After the flow comes to a halt, a depth $h_{\text {stop }}$ of static grains remains over the geotextile. This quantity resembles similar residuals on inclined granular flows over a bumpy base $[4,16]$ or felt cloth [28], which exist in a range of inclinations, and decrease with $\tan \alpha$. Here Fig. 4 shows that the data conform to the least-squares fit

$$
h_{\text {stop }}^{\dagger} \equiv h_{\text {stop }} / \bar{d}=a_{h} /\left(\tan \alpha-\tan \alpha_{0}\right)
$$




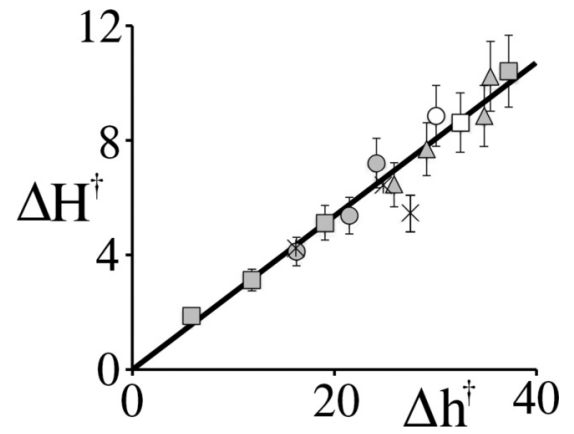

FIG. 5. Dimensionless mass hold-up $\Delta H^{\dagger}=H^{\dagger}-H_{\text {stop }}^{\dagger}$ in the flowing layer vs dimensionless flow depth $\Delta h^{\dagger}=h^{\dagger}-h_{\text {stop. Symbols }}^{\dagger}$. S conform to inclination angles shown in Fig. 4. Error bars are $95 \%$ confidence intervals. The slope is the mean solid volume fraction $\bar{v}$ in Eq. (27). The error bar at $\alpha=31^{\circ}$ ( $\times$ symbol) is an estimate based on errors calculated at other inclinations.

where $a_{h}=0.653 \pm 0.053$ and $\tan \alpha_{0}=0.5715 \pm 0.0027$. However, as with flows over a bumpy base $[21,35,36], h_{\text {stop }}$ is likely to depend on the energy dissipation characteristics of the basal boundary, and thus it is not, strictly speaking, a length scale of the flow itself. Moreover, for a natural flow over a deep pile, $h_{\text {stop }}$ is indefinite.

Although grains undergo creeping motion at distances below $\left(h-h_{\text {stop }}\right)$ from the free surface $[25,37]$, this difference between overall flow thickness and its residual at rest is a representative scale for the depth of the flowing layer, as the measurements of Ref. [38] suggested. Therefore, we explore how mass flow rate and speed scale with

$$
\Delta h \equiv h-h_{\text {stop }},
$$

and we refer to this difference as the "flow depth." Inspired by Eq. (21), we define the corresponding change in mass holdup as

$$
\Delta H \equiv H-H_{\text {stop }} .
$$

A reduction of geotextile strain data further reveals that mass hold-up $\Delta H$ in the flowing layer is indeed proportional to $\Delta h$ for inclinations steeper than the angle of repose $\alpha_{r}$ (Fig. 5). In that figure, mass hold-up and depth are made dimensionless through

$$
H^{\dagger} \equiv H /\left(\rho_{s} \bar{d}\right)
$$

and

$$
h^{\dagger} \equiv h / \bar{d}
$$

As Eq. (21) suggests, Fig. 5 implies that the mean volume fraction in the flowing layer is

$$
\bar{v}=0.268 \pm 0.033,
$$

irrespective of flow conditions. At $\alpha=31^{\circ}$ below the angle of repose ( $\times$ symbol), a steady, fully developed flow is difficult to establish, making it more challenging to exploit the FBG signal. However, at that angle, the data suggest $\bar{v} \simeq 0.20$.

The mean volume fraction in Eq. (27) is similar to that obtained in Ref. [25] by integrating depth profiles of $v$ in numerical simulations of steeper granular flows over erodible "sidewall-stabilized heaps" (SSHs) confined between nearby

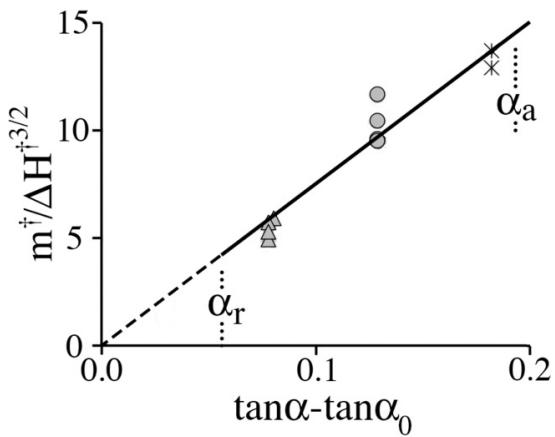

FIG. 6. Dimensionless discharge mass flow rate [Eq. (28)] relative to the $3 / 2$ power of dimensionless hold-up in the flowing layer vs excursion in $\tan \alpha$ beyond its jamming value $\tan \alpha_{0}$ for $\alpha_{r}<\alpha<\alpha_{a}$. The solid line is Eq. (29). The vertical dotted lines mark angles of repose $\alpha_{r}$ and spontaneous avalanching $\alpha_{a}$.

plates with $0.6<W / \Delta h<40$. Because our own side walls are more distant relative to the flow thickness, and because, as we will later establish, the flow comes to rest well above the geotextile, low mean volume fractions in the flowing layer transcend the SSH phenomenon and are likely associated with the dissipative nature of grains at rest below the flow, rather than with the presence of side walls or geotextile.

Figures 6 and 7 indicate how the discharge mass flow rate $\dot{m}$ varies with, respectively, angle of inclination and mass hold-up in the flowing layer in a steady flow. Making it dimensionless as

$$
\dot{m}^{\dagger} \equiv \frac{\dot{m}}{\rho_{s} W \bar{d} \sqrt{g \bar{d}}}
$$

we find, for $\alpha>\alpha_{r}$,

$$
\dot{m}^{\dagger}=a_{m} \Delta H^{\dagger 3 / 2}\left(\tan \alpha-\tan \alpha_{0}\right),
$$

where $a_{m}=75.0 \pm 4.6$. This dependence of mass flow rate on the $3 / 2$ power of the flow depth is in sharp contrast with its $5 / 2$ counterpart for flows on a rigid bumpy boundary.

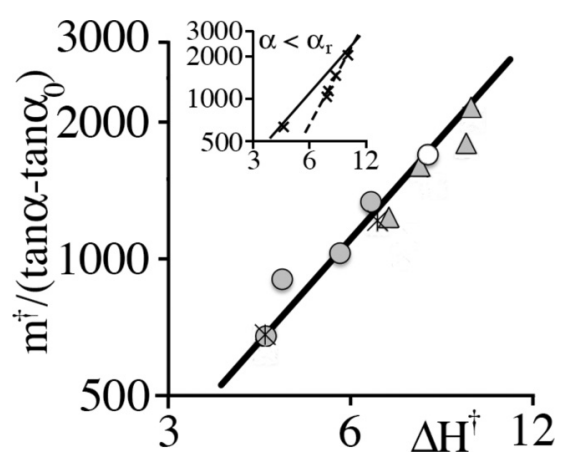

FIG. 7. Dimensionless discharge mass flow rate [Eq. (28)] relative to $\left(\tan \alpha-\tan \alpha_{0}\right)$ vs dimensionless hold-up in the flowing layer. Symbols conform to inclination angles shown in Figs. 4 and 6. The main graph is for inclinations steeper than the angle of repose $\alpha_{r}$. The inset is for $\alpha=31^{\circ}<\alpha_{r}$. The solid line is inclined at a 3/2 slope [Eq. (29)] and the dashed line at 5/2 [Eq. (34)]. 


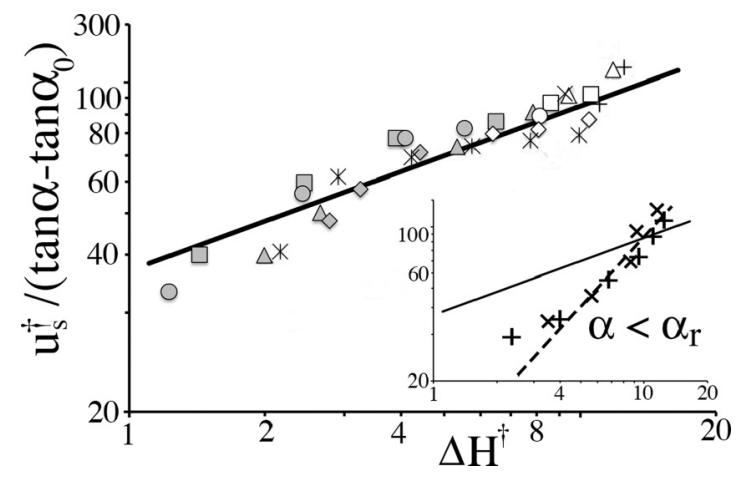

FIG. 8. Dimensionless surface velocity [Eq. (30)] relative to $\left(\tan \alpha-\tan \alpha_{0}\right)$ vs dimensionless hold-up in the flowing layer. Symbols conform to inclination angles shown in Figs. 4-7. The main graph is for inclinations steeper than the angle of repose $\alpha_{r}$, while the inset is for $\alpha=31.5^{\circ}(\times)$ and $32^{\circ}(+)$, both $<\alpha_{r}$. Solid lines are inclined at a $1 / 2$ slope [Eq. (31)] and the dashed line at 3/2 [Eq. (35)].

Consistent with Eq. (28), we make all velocities dimensionless with $\sqrt{g \bar{d}}$,

$$
u^{\dagger} \equiv u / \sqrt{g \bar{d}}
$$

Then, as Fig. 8 shows, independent measurements of the surface velocity $u_{s}$ for inclinations steeper than the angle of repose $\alpha_{r}$ scale as

$$
u_{s}^{\dagger}=a_{u} \Delta H^{\dagger 1 / 2}\left(\tan \alpha-\tan \alpha_{0}\right),
$$

where $a_{u}=53.5 \pm 2.8$. Equivalently, this suggests that the Froude number based on surface velocity and holdup in the flowing layer,

$$
\operatorname{Fr}_{s} \equiv \frac{u_{s}^{\dagger}}{\sqrt{\Delta H^{\dagger}}}
$$

grows linearly with $\left(\tan \alpha-\tan \alpha_{0}\right)$ for $\alpha>\alpha_{r}$.

$$
\mathrm{Fr}_{s}=a_{u}\left(\tan \alpha-\tan \alpha_{0}\right) .
$$

However, as the inset of Fig. 7 shows, the scaling of mass flow rate in Eq. (29) is no longer valid at inclinations $\alpha=31.5^{\circ}$ and $32^{\circ}$ below the angle of repose. Here a more appropriate dependence near arrest of the flow is

$$
\dot{m}^{\dagger}=a_{m}^{\prime} \Delta H^{\dagger 5 / 2}\left(\tan \alpha-\tan \alpha_{0}\right),
$$

where $a_{m}^{\prime}=7.4 \pm 1.2$. At such low inclinations $<\alpha_{r}$, the inset of Fig. 8 also suggests that surface velocity scales as

$$
u_{s}^{\dagger}=a_{u}^{\prime} \Delta H^{\dagger 3 / 2}\left(\tan \alpha-\tan \alpha_{0}\right),
$$

with $a_{u}^{\prime}=5.38 \pm 0.61$ or, equivalently,

$$
\mathrm{Fr}_{s}=a_{u}^{\prime} \Delta H^{\dagger}\left(\tan \alpha-\tan \alpha_{0}\right)
$$

which resembles the Froude dependence in Eq. (3) of Deboeuf et al. [38].

Unlike grains discharged upon narrower heaps [10], our distant side walls did not visibly affect the relatively shallow flows that formed on this highly dissipative base. In typical experiments, the overhead camera recorded a uniform velocity in the transverse direction over $>90 \%$ of the channel width. To confirm this apparent lateral invariance of the velocity, we

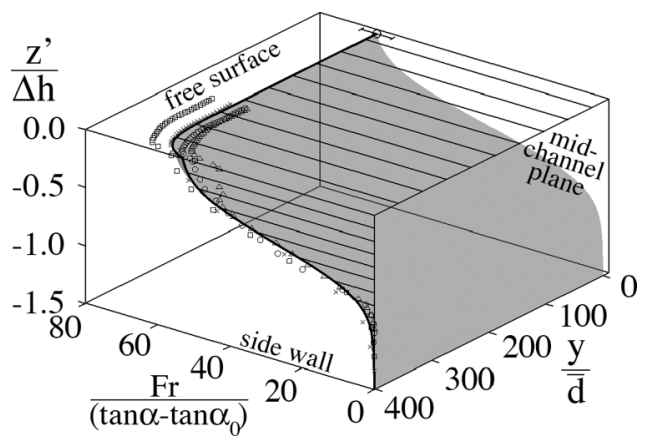

FIG. 9. Profile of local dimensionless velocity $\mathrm{Fr} \equiv u^{\dagger} / \sqrt{\Delta H^{\dagger}}$ over one half of the channel relative to $\left(\tan \alpha-\tan \alpha_{0}\right)$ vs dimensionless lateral distance $y / \bar{d}$ from the channel center plane and vs dimensionless distance $\zeta \equiv z^{\prime} / \Delta h$ from the free surface, counted positive upward. The symbols $\times, \square$, $\circ$, and $\diamond$ correspond to measurements at $\Delta H^{\dagger}=2.4,3.9,6.5$, and 8.6, respectively. The solid line is a best fit $\mathrm{Fr}=\left(\tan \alpha-\tan \alpha_{0}\right)\left[1+\tanh \left(2+4 z^{\prime} / \Delta h\right)\right]\left\{u_{c}^{\dagger}-\left(u_{c}^{\dagger}-u_{w}^{\dagger}\right)\right.$ $\exp [-(W / 2-y) / \ell]\} /(1+\tanh 2)$, and the gray surface plot is its extrapolation throughout the flow. Lines parallel to the Fr axis represent velocity vectors. For these measurements at $\alpha=34^{\circ}>\alpha_{r}$, the surface profiles have $u_{c}^{\dagger}=63 \pm 7$ (represented by an open circle and its error bar on the midplane), $u_{w}^{\dagger}=53 \pm 6$, and $\ell / \bar{d}=$ $21.3 \pm 0.9$.

trained the Photron high-speed camera on a narrower region of the free surface within $5 \mathrm{~cm}$ of a side wall. We then moved the same high-speed camera to observe depth profiles through the transparent wall.

Figure 9 patches the two resulting velocity profiles in dimensionless form for several flow thicknesses. Our profiles along depth have a concavity that is inverted from that on a rigid base [4,5,21], but resembles similar concavity for dissipative bases like the SSH [25] or erodible beds of lower inclination [10].

In the transverse direction, we found that the surface velocity profile has a similar exponential form to that recorded in Ref. [10], namely,

$$
u_{s} \simeq u_{w}+\left(u_{c}-u_{w}\right)\{1-\exp [-(W / 2-|y|) / \ell]\},
$$

where $u_{w}$ and $u_{c}$ are, respectively, velocities at the wall and on the channel centerline, and $[(W / 2)-|y|]$ is lateral distance from the wall. Although the apparatus in Ref. [10] did not fix mass flow rate and inclination independently, these authors observed $2<\ell / \bar{d}<49$ for $19 \leqslant W / \bar{d} \leqslant 570$ at the single discharge mass flow rate $\dot{m} \simeq 89$. In terms of side wall distance, their ratio $\ell / W$ first increased with $\tan \alpha$ for $22^{\circ}<\alpha \lesssim 24^{\circ}$ to a maximum $\ell / W \simeq 0.13$, but then decreased from $24^{\circ}$ to $32.5^{\circ}$, thus suggesting that transverse velocity profiles become more uniform at steeper inclinations, consistent with observations in the SSH $[9,26]$. In general, the range of length scales $2<\ell / \bar{d}<49$ reported in Ref. [10] with glass beads suggests that confinement only affects the transverse velocity profile within a narrow wall region, which, in our case, constitutes a negligible fraction of the channel width. In short, at the inclinations and relatively wide channel that we staged, side walls barely affected transverse velocity profiles. 
However, as SSH experiments showed, this observation does not necessarily imply that the overall streamwise force balance is insensitive to side walls. To establish whether or not our side walls matter to this balance, we calculate an upper bound for the ratio of side wall friction $F_{w}$ to weight $P$ projected along $x$ on a cross section of unit length through the entire flowing layer of width $W$ and height $\Delta h$, assuming that a constant friction coefficient $\mu_{w}$ converts the normal stress $N$, which grows with depth, to $F_{w}$. Because normal stress and friction force satisfy $-d N / d z^{\prime}=\rho_{s} v g \cos \alpha<\rho_{s} v_{c} g \cos \alpha_{0}$ and $d F_{w} / d z^{\prime}=\mu_{w} N$, the latter integrates to the upper bound $F_{w}<\mu_{w} \rho_{s} v_{c} g \cos \alpha_{0} \Delta h^{2} / 2$. Meanwhile, the component of the flowing layer weight along $x$ is $P=\rho_{s} \bar{v} \Delta h W g \sin \alpha>$ $\rho_{s} \bar{v} \Delta h W g \sin \alpha_{0}$. Therefore, the ratio of side wall friction to weight is, at worst, $F_{w} / P<\mu_{w} v_{c} \Delta h /\left(2 \bar{v} W \tan \alpha_{0}\right)$. With a typical wall friction $\mu_{w} \sim 0.5$ [9], our upper bound in flow thickness $\Delta h<40 \bar{d}$ and large width $W \simeq 805 \bar{d}$ yields $F / P<5 \%$. Therefore, unlike the SSH $[9,25,26]$, our side wall friction hardly contributes to the force balance.

Although our side walls barely affect the overall force balance or transverse profiles of streamwise velocity, they channel the flow, thereby letting us stage arbitrary depths. In natural flows without such confinement, an increase in mass flow rate is typically met with greater width, while depth barely changes [16].

As Fig. 9 shows, velocity vanishes within experimental error at $\left|z^{\prime}\right|=\gamma \Delta h$, where $\gamma \simeq 1.2$. Therefore, the nature of the geotextile should be of little consequence, as long as the free surface of elevation $h$ is sufficient to contain the flow thickness $\Delta h$ or, equivalently, whenever $h / h_{\text {stop }}<\gamma /(\gamma-$ $1) \simeq 6$. Most of our experiments satisfied this condition. Those that did not are shown as open symbols in Figs. 5-8. Because these flows of relatively large thickness did not produce abnormal data, the geotextile was a meaningful surrogate for dissipative sand heaps, as proposed in Ref. [28].

Finally, if we ignore distant side walls, it is instructive to compare the form of our dimensionless mass flow rates and surface velocities, which differ by a factor of $\Delta H^{\dagger}$ for inclinations above the angle of repose $\alpha_{r}$ [Eqs. (29) and (31)] or below $\alpha_{r}$ [Eqs. (34) and (35)]. As Richard et al. [25] noted in simulations of SSH flows, depth profiles of velocity and volume fraction can be made to collapse into universal functions of the relative depth coordinate $\zeta \equiv z^{\prime} / \Delta h$ that no longer depend on flow depth and inclination, if the latter is steep enough to avoid jamming. In similar observations, our own velocity profiles with $\alpha>\alpha_{r}$ confirm invariance of the Froude number with $\Delta H^{\dagger}$ (Fig. 9) or, equivalently, of the ratio $u / u_{s}$ with $\Delta H^{\dagger}$. Then, if volume fraction depth profiles $v(\zeta)$ are independent of flow thickness $\Delta h$, and if velocity relative to surface speed $u_{s}$ has a form $u / u_{s} \equiv \phi(\zeta)$ that is also independent of $\Delta h$, then the mass flow rate must integrate to

$$
\dot{m}=\rho_{s} u_{s} W \Delta h \int_{-\infty}^{0} v(\zeta) \phi(\zeta) d \zeta
$$

or, substituting the result in Eq. (27)

$$
\dot{m}^{\dagger}=a_{I} u_{s}^{\dagger} \Delta H^{\dagger} / \bar{v},
$$

where $a_{I}$ is a constant equal to the integral in Eq. (38). Comparing Eqs. (29) and (31) for $\alpha>\alpha_{r}$, our results then imply $a_{I}=a_{m} \bar{v} / a_{u}=0.376 \pm 0.036$. In short, above the angle of repose, the profiles in Fig. 9 and the independent scalings for $\dot{m}^{\dagger}$ and $u_{s}^{\dagger}$ suggest that both $v$ and $u / u_{s}$ vary only with relative distance $\zeta=z^{\prime} / \Delta h$. For inclinations below the angle of repose, Eqs. (34) and (35) and the data point $(x$ symbol) at $\alpha=31^{\circ}$ yield a similar integral $a_{I}=0.27 \pm 0.06$. However, it remains to confirm with additional velocity measurements at inclinations $<\alpha_{r}$ near jamming whether depth velocity profiles $u / u_{s}$ also remain invariant with flow thickness.

\section{CONCLUSIONS}

By reconciling simultaneous measurements of mass flow rate, surface speed, flow depth, and mean solid volume fraction on steady flows of grains in a wide inclined channel, we confirmed that a dissipative base supports different flows than a rigid base, whether flat or bumpy. We replicated the behavior of an erodible granular heap using the technique in Ref. [28], where a felt cloth was laid on an inclined surface to dissipate fluctuation energy. We refined this method by embedding a fiber Bragg grating in a composite geotextile of known elasticity. Our analysis of the resulting signal showed that the weight of flowing material could be inferred from the difference in steady microstrain during flow and after grains settled into a residual layer at rest. Combined with the corresponding measurements of flow depth, we calculated a relatively low value of mean solid volume fraction $\bar{v}=0.268 \pm 0.033$, independent of discharge rate or inclination, consistent with experiments [19] or numerical simulations [25] with steeper, side wall-dominated SSH flows on dissipative heaps in narrower channels. Side walls affected only the transverse profiles of surface velocity within a short distance relative to the channel width $W$. Moreover, a worstcase estimate revealed that, at the relatively large $W / \bar{d} \simeq 805$ of our experiments, side wall friction was small compared to the streamwise component of weight in the flowing layer.

Our experiments showed that, when grains are forced upon an erodible slope, flows can be sustained at inclinations less than the angle of repose $\alpha_{r}$, as long as these remain above the asymptotic value $\alpha_{0}$. In the terminology of Ref. [28], $\alpha_{a}$ and $\alpha_{0}$ correspond, respectively, to the asymptotic inclinations where $h_{\text {start }}$ and $h_{\text {stop }} \rightarrow \infty$, i.e., where the heap is sufficiently deep to be independent of the nature of the base upon which it rests.

At inclinations $\alpha_{r}<\alpha<\alpha_{a}$ steeper than the angle of repose, but smaller than the angle of spontaneous avalanching, we found that mass flow rate grows with $\left(\tan \alpha-\tan \alpha_{0}\right)$ and the $3 / 2$ power of the mass holdup. The corresponding surface velocity has a compatible scaling, producing a Froude number that is only proportional to $\left(\tan \alpha-\tan \alpha_{0}\right)$. Our velocity profiles measurements along depth also implied that velocity relative to its value at the surface is a universal function of the depth coordinate relative to the flow thickness. At inclinations $\alpha_{0}<\alpha<\alpha_{r}$ below the angle of repose, we found that the mass flow rate scales instead as the $5 / 2$ power of the mass holdup. 


\section{ACKNOWLEDGMENTS}

We are grateful to Jean-Pierre Giroud and Anil Netravali for advice on geotextiles, to Patrick Chasle and Cheikh Fall for help with experiments, to Jim McElwaine for an illuminating discussion on inclined flows, and to Philippe Boltenhagen for measuring $v_{c}, \alpha_{r}$, and $\alpha_{a}$. This paper was made possible by the support of NPRP grant 6-059-2-023 from the Qatar National Research Fund.
[1] P. C. Johnson, P. Nott, and R. Jackson, J. Fluid Mech. 210, 501 (1990).

[2] A. J. Holyoake and J. N. McElwaine, J. Fluid Mech. 710, 35 (2012).

[3] D. M. Hanes and O. R. Walton, Powder Tech. 109, 133 (2000).

[4] O. Pouliquen, Phys. Fluids 11, 542 (1999).

[5] L. E. Silbert, D. Ertaş, G. S. Grest, T. C. Halsey, D. Levine, and S. J. Plimpton, Phys. Rev. E 64, 051302 (2001).

[6] J.-P. Bouchaud, M. Cates, J. R. Prakash, and S. F. Edwards, J. Phys. I France 4, 1383 (1994).

[7] T. Boutreux, E. Raphaël, and P.-G. de Gennes, Phys. Rev. E 58, 4692 (1998).

[8] D. V. Khakhar, A. V. Orpe, P. Andresén, and J. M. Ottino, J. Fluid Mech. 441, 255 (2001).

[9] N. Taberlet, P. Richard, A. Valance, W. Losert, J. M. Pasini, J. T. Jenkins, and R. Delannay, Phys. Rev. Lett. 91, 264301 (2003).

[10] P. Jop, Y. L. Forterre, and O. Pouliquen, J. Fluid Mech. 541, 167 (2005).

[11] A. de Ryck, H. P. Zhu, S. M. Wu, A. B. Yu, and P. Zulli, Powder Tech. 203, 125 (2010).

[12] A. Mangeney, O. Roche, O. Hungr, N. Mangold, G. Faccanoni, and A. Lucas, J. Geophys. Res. 115, F03040 (2010).

[13] B. P. Kokelaar, R. L. Graham, J. M. N. T. Gray, and J. Vallance, Earth Planet. Sci. Lett. 385, 172 (2014).

[14] B. Andreotti, P. Claudin, and S. Douady, Eur. Phys. J. B 28, 321 (2002).

[15] G. Félix and N. Thomas, Earth Planet. Sci. Lett. 221, 197 (2004).

[16] D. Takagi, J. N. McElwaine, and H. E. Huppert, Phys. Rev. E 83, 031306 (2011).

[17] C. G. Johnson, B. P. Kokelaar, R. M. Iverson, M. Logan, R. G. LaHusen, and J. M. N. T. Gray, J. Geophys. Res. 117, F01032 (2012).

[18] N. Mangold, A. Mangeney, V. Migeon, V. Ansan, A. Lucas, D. Baratoux, and F. Bouchut, J. Geophys. Res. 115, E11001 (2010).
[19] R. Delannay, M. Louge, P. Richard, N. Taberlet, and A. Valance, Nat. Materials 6, 99 (2007).

[20] D. Berzi and J. T. Jenkins, Phys. Fluids 23, 013303 (2011).

[21] M. Y. Louge, Phys. Rev. E 67, 061303 (2003).

[22] M. Y. Louge and S. C. Keast, Phys. Fluids 13, 1213 (2001).

[23] N. Brodu, P. Richard, and R. Delannay, Phys. Rev. E 87, 022202 (2013).

[24] N. Brodu, R. Delannay, A. Valance, and P. Richard, J. Fluid Mech. 769, 218 (2015).

[25] P. Richard, A. Valance, J.-F. Metayer, P. Sanchez, J. Crassous, M. Louge, and R. Delannay, Phys. Rev. Lett. 101, 248002 (2008).

[26] W. Bi, R. Delannay, P. Richard, N. Taberlet, and A. Valance, J. Phys.: Condens. Matter 17, S2457 (2005).

[27] J. R. L. Allen, J. Geol. 78, 326 (1970).

[28] A. Daerr and S. Douady, Nature (London) 399, 241 (1999).

[29] Y.-J. Rao, Meas. Sci. Technol. 8, 355 (1997).

[30] M. Creyssels, P. Dupont, A. Ould el Moctar, A. Valance, I. Cantat, J. T. Jenkins, J. M. Pasini, and K. R. Rasmussen, J. Fluid Mech. 625, 47 (2009).

[31] H. Ahn, C. E. Brennen, and R. H. Sabersky, J. Appl. Mech. 58, 792 (1991).

[32] K. Platzer, P. Bartelt, and M. Kern, Geophys. Res. Lett. 34, L07501 (2007).

[33] P. G. Rognon, F. Chevoir, H. Bellot, F. Ousset, M. Naaïm, and P. Coussot, J. Rheol. 52, 729 (2008).

[34] A. Nancey, B. Lacina, and J. Henderson, in Geotextile and Optic Fibers: Feedback after Four Years of Use in Soil, Geosynthetics 2007, GRI-20, Washington D.C., USA (Geosynthetic Materials Association, Roseville, MN, 2007).

[35] V. Kumaran, J. Fluid Mech. 599, 121 (2008).

[36] S. Maheshwari and V. Kumaran, Granular Matter 14, 209 (2012).

[37] T. S. Komatsu, S. Inagaki, N. Nakagawa, and S. Nasuno, Phys. Rev. Lett. 86, 1757 (2001).

[38] S. Deboeuf, E. Lajeunesse, O. Dauchot, and B. Andreotti, Phys. Rev. Lett. 97, 158303 (2006). 